\title{
Luminosity dependent covering factor of the dust torus around AGN viewed with AKARI and WISE
}

\author{
Yoshiki Toba*1,2, Shinki Oyabu ${ }^{3}$, Hideo Matsuhara ${ }^{2}$, Matthew A. Malkan ${ }^{4}$, Daisuke \\ Ishihara $^{3}$, Takehiko Wada $^{2}$, Youichi Ohyama ${ }^{5}$, Satoshi Takita ${ }^{2}$, and Chisato \\ Yamauchi $^{6}$ \\ ${ }^{1}$ The Graduate University for Advanced Studies (SOKENDAI), Japan \\ ${ }^{2}$ Institute of Space and Astronautical Science, Japan Aerospace Exploration Agency, Japan \\ ${ }^{3}$ Graduate School of Science, Nagoya University, Japan \\ ${ }^{4}$ Department of Physics and Astronomy, University of California, USA \\ ${ }^{5}$ Institute of Astronomy and Astrophysics, Taiwan \\ ${ }^{6}$ Astronomy Data Center, National Astronomical Observatory of Japan, Japan \\ E-mail: tobadir.isas.jaxa.jp
}

\begin{abstract}
We present the relation between the covering factor (CF) of active galactic nuclei (AGNs) and mid-infrared (MIR) luminosity from the $22 \mu \mathrm{m}$ luminosity functions (LFs) using the Wide-field Infrared Survey Explorer (WISE). Combining the WISE catalog with the Sloan Digital Sky Survey (SDSS) spectroscopic catalog, $\sim 30,000$ galaxies were selected. We then extracted $\sim 5,000$ AGNs from them according to the optical emission lines and catalog classification in the SDSS. We found CF decreases with MIR luminosity. This result supports the findings of $18 \mu \mathrm{m} \mathrm{LFs}$ obtained by the Japanese astronomical satellite, AKARI.
\end{abstract}

Nuclei of Seyfert galaxies and QSOS - Central engine \& conditions of star formation November 6-8, 2012

Max-Planck-Insitut für Radioastronomie (MPIfR), Bonn, Germany

\footnotetext{
* Speaker.
} 


\section{Introduction}

Active galactic nuclei (AGNs) are among the most interesting objects in the universe. Although there are numerous AGN classes, a unified scheme has been emerging steadily (Eq. []]). This scheme proposes a geometrically thick dusty torus surrounds the AGN central engine. The torus provides anisotropic obscuration of the central region so that sources viewed face-on are recognized as type 1 AGN and those observed edge-on are type 2 AGNs. Therefore, the basic premise of the scheme is that all AGNs are fundamentally the same: accreting supermassive black holes. However, the key parameters of dust torus unification such as its intrinsic geometry and physical properties are still unclear. In this work, we focus on the covering fraction of the dust torus. The covering factor (CF) is defined as the fraction of the sky seen from the AGN center which is blocked by obscuring material. This quantity is one of the most important physical parameter for characterizing the torus structure. Recently, some authors have reported the CF depends on the luminosity (Eq. [目],[[2]). On the other hand, some authors questioned these dependences, claiming that these results are affected by various uncertainties (Eq. [G], [可]). In particular, these observed correlations can be explained as a selection effect, in which case they may not necessarily have astrophysical significance. To resolve this question, infrared (IR) observations of AGNs are important, because the reprocessed radiation from the dust in the torus is re-emitted in this wavelength range. In this work, we use the mid-IR (MIR) luminosity function (LF). The AGN LF is a statistical tool for characterizing the properties of the CF of AGN populations. By using the LF, we can discuss the luminosity dependence statistically. Throughout this paper, we assume a flat Universe with $\Omega_{k}=0$, and we adopt $\left(\Omega_{M}, \Omega_{\Lambda}\right)=(0.3,0.7)$ and $H_{0}=70 \mathrm{~km} \mathrm{~s}^{-1} \mathrm{Mpc}^{-1}$.

\section{Method}

\subsection{Sample selection}

The Wide-field Infrared Survey Explorer (WISE) performed MIR all-sky survey with 3.4, 4.6, 12 , and $22 \mu \mathrm{m}$ [ए]]. Its spatial resolution and sensitivity are much better than those of the IRAS and AKARI which performed previous IR all-sky survey. Our sample is selected from the WISE sources with spectroscopy from the Sloan Digital Sky Survey (SDSS) Data Release 8 [W]. Figure W shows the flow chart of the sample selection. First, our sample was obtained from the 82,107,768 WISE sources in the SDSS spectroscopic region. These objects were then cross matched with the SDSS spectroscopic catalog. Next, we extracted objects with $z>0.006$ from the sample. This criterion is to exclude stars and nearby galaxies. After that, to ensure flux accuracy, we extracted objects with 12 and $22 \mu \mathrm{m}$ flux higher than 0.9 and $9.0 \mathrm{mJy}$, respectively. The flux limits with a completeness of almost $100 \%$ were calculated from the Explanatory Supplement to the WISE AllSky Data Release Products [四]. Finally, about 300,000 for $12 \mu \mathrm{m}$ and 30,000 for $22 \mu \mathrm{m}$ galaxies have been selected through these steps.

\subsection{Type classification}

Next, we have classified these galaxies into five types using optical line flux as shown in Figure ․ First, we separated Seyfert 1 galaxies (Sy1, hereafter type 1 AGN) from narrow line galaxies, by referring to the SDSS spectroscopic type. Then, after rejecting those with low significance for Ha, 


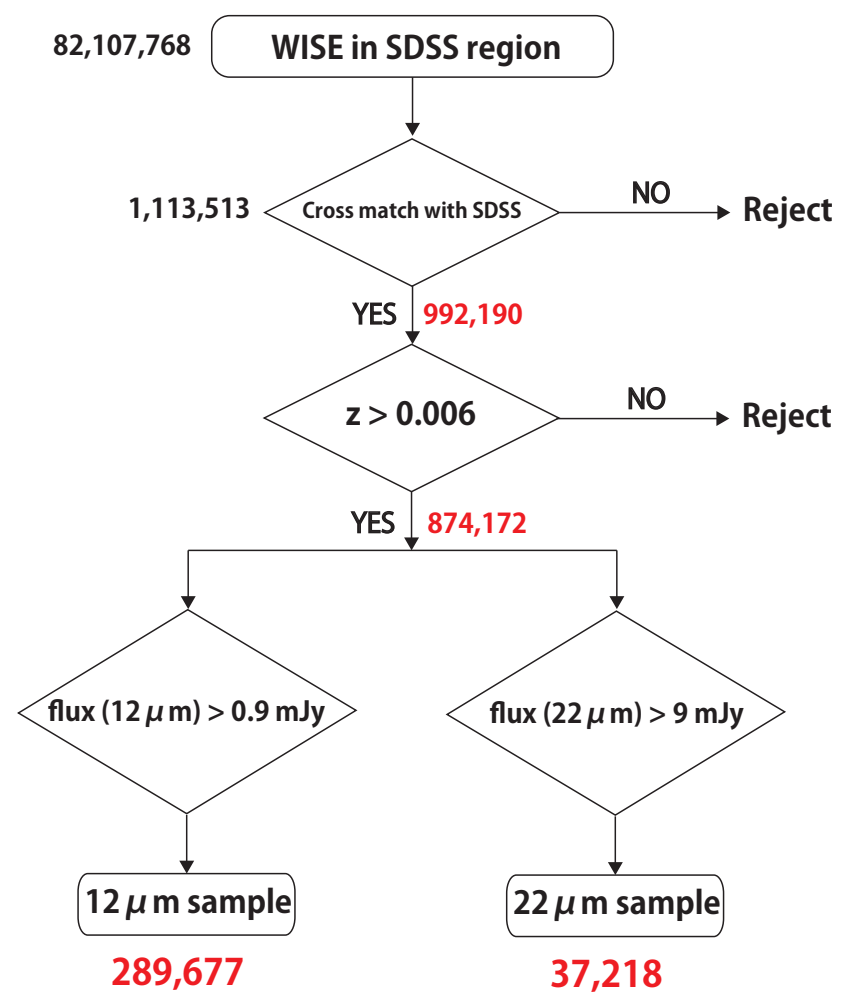

Figure 1: The flow chart of a process for sample selection.

$\mathrm{Hb},[\mathrm{OII}]$, and $[\mathrm{NII}]$ emission lines, the remaining narrow line galaxies were classified into Seyfert 2 galaxies (Sy2), low ionization narrow emission line galaxies (LINER), galaxies that are likely to contain both star formation and AGN activity (composite types of galaxies, hereinafter Composite), and star-forming galaxies (SF) from optical emission line ratios of $[\mathrm{OIII}] / \mathrm{H} \beta$ and $[\mathrm{NII}] / \mathrm{H} \alpha$ (it is called BPT diagram. see [B]]).

\section{Result}

\subsection{The luminosity functions at 12 and $22 \mu \mathrm{m}$}

Here we obtain the luminosity functions (i.e. the volume density of galaxies per unit magnitude range) of WISE-SDSS galaxies. The LFs are derived by following the $1 / V_{\max } \operatorname{method}([\mathbb{W}])$. The volume density $\phi(L)$ and its uncertainty $\sigma_{\phi(L)}$ were derived using the expressions:

$$
\begin{gathered}
\phi(L)=\sum_{i}^{N} \frac{1}{V_{\max , i}}, \\
\sigma_{\phi(L)}=\sqrt{\sum_{i}^{N} \frac{1}{V_{\max , i}^{2}},}
\end{gathered}
$$

where $V_{\max }$ is the maximum co-moving volume of $i$ th galaxies after applying K-correction, and the sum is over all galaxies in each luminosity bin. 


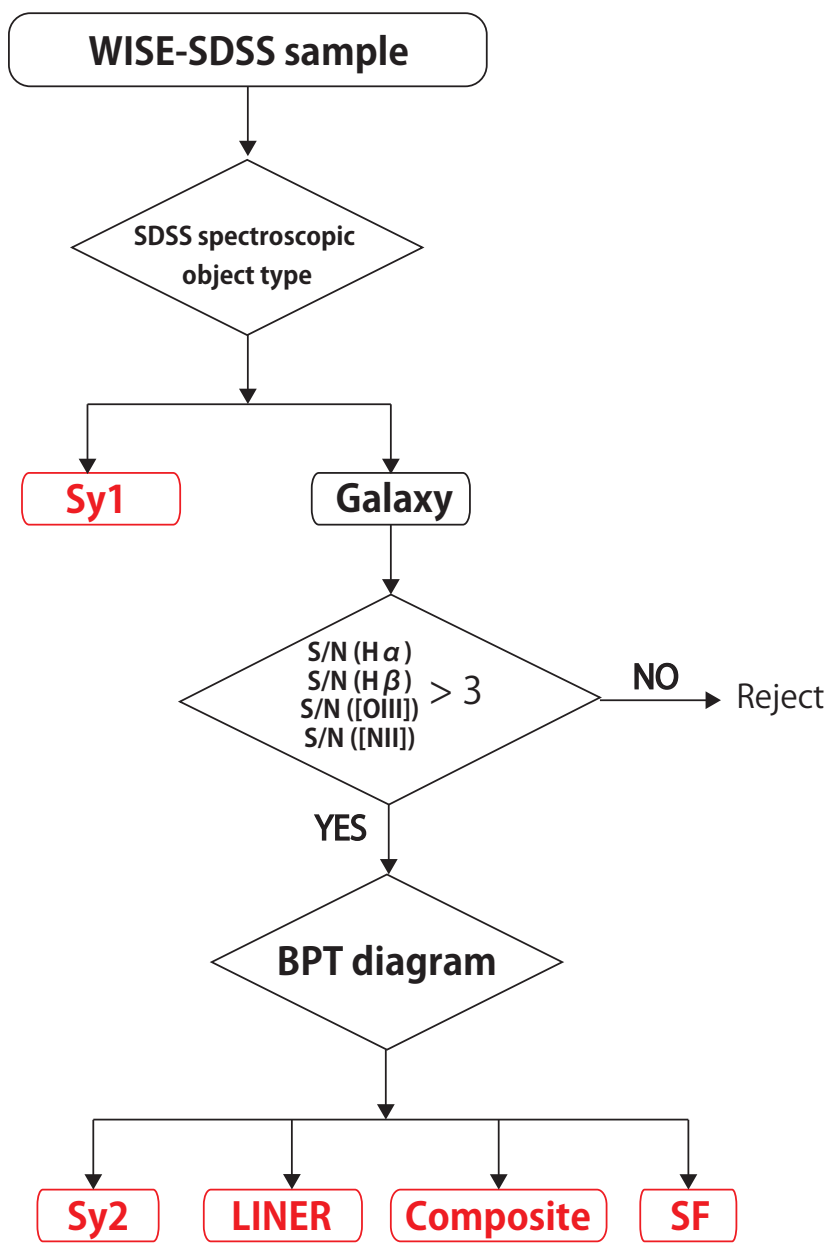

Figure 2: The flow chart of type classification.

Figure [3] shows the LFs at 12 and $22 \mu \mathrm{m}$ for each spectroscopic type of galaxy. The SFs are the most numerous objects at low luminosities, while the AGNs dominate the volume density at luminosities above $\sim 10^{10} L_{\odot}$. This tendency was also reported by [प]] based on their $12 \mu \mathrm{m}$ fluxlimited sample using the IRAS Faint Source Catalogue (FSC), and by [[13] based on 9 and $18 \mu \mathrm{m}$ galaxies using the AKARI MIR all-sky survey catalogue [प].

\section{Discussion}

\subsection{Luminosity dependence of covering factor}

We estimated the CF in order to constrain the structure of the hypothesized dust torus invoked by unification. Mathematically, CF for a certain luminosity range can be described the fraction of type 2 AGNs out of entire AGN population as shown by this formula,

$$
C F=\frac{\Phi_{2}}{\Phi_{1}+\Phi_{2}},
$$

where, $\Phi_{1}$ and $\Phi_{2}$ represent the number density for type 1 and type 2 AGNs, respectively. In this work, we assume that the MIR luminosity of type 1 and type 2 AGNs should be intrinsically 


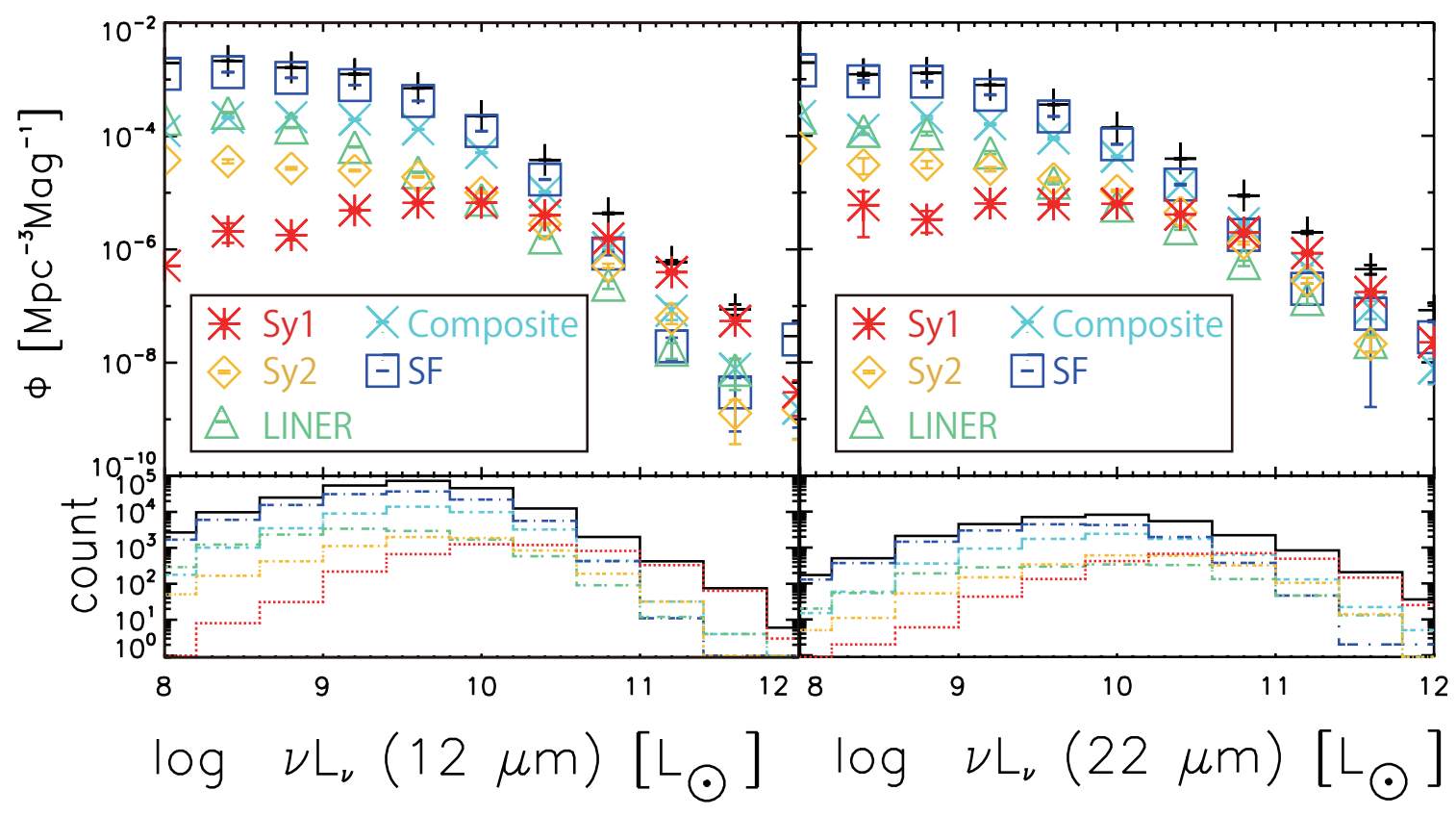

Figure 3: The LFs at 12 (left) and 22 (right) $\mu \mathrm{m}$ for each type of galaxy, plotted in terms of space density as a function of luminosity. The error bars are calculated from Poisson-statistical uncertainty.

the same. By integrating the LFs of type 1 and type 2 AGNs separately, we obtained the number density $\Phi$ for each AGN type, which is given by the following formula:

$$
\Phi=\int_{L} \phi(L) \mathrm{d} L \sim \sum_{i} \phi_{i}(L) \Delta L .
$$

Note that in this work, we basically consider Sy2 as type 2 AGNs. However, galaxies exhibiting type-2 AGN like properties are possibly included among LINERs and Composites. So, we also estimate the covering factor including these possibilities. Figure $\mathbb{\theta}$ shows the fraction of type 2 AGNs, representing the CF of AGN torus as a function of MIR luminosity. As shown in Figure $\mathbb{\text { H, }}$ CF decreases with MIR luminosity regardless of type 2 AGN classification. In the Figure, more than 5000 AGNs in total are plotted. Therefore, our result is statistically robust compared with previous studies. There WISE results strongly support our previous result obtained by AKARI [ए]3].

\section{References}

[1] Aihara, H., Allende Prieto, C., An, D., et al. 2011, A\&AS 193, 29

[2] Antonucci, R. 1993, ARA\&A 31, 473

[3] Baldwin, J.A., Phillips, M.M., \& Terlevich, R. 1981, PASP 93, 5

[4] Cutri, R.M., Wright, E.L., Conrow, T., et al. 2012, Explanatory Supplement to the WISE All-Sky Data Release Products

[5] Dwelly, T., \& Page, M.J. 2006, MNRAS 372, 1755

[6] Hasinger, G. 2008, A\&A 490, 905 


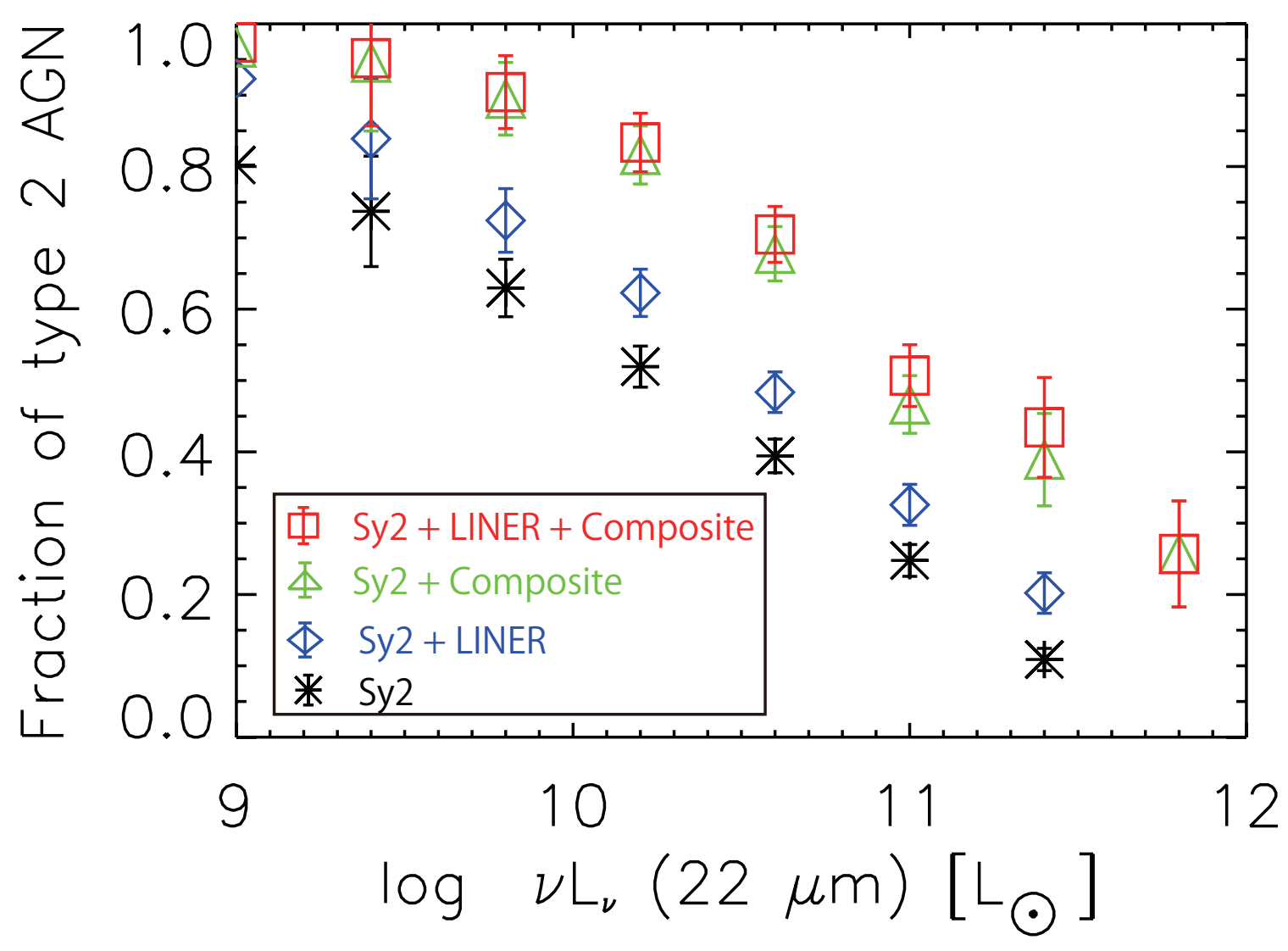

Figure 4: The fraction of type $2 \mathrm{AGN}$ as a function of $22 \mu \mathrm{m}$ luminosity. Each symbol represents a different possible assumption about which populations were included as type 2 AGNs.

[7] Ishihara, D., Onaka, T., Kataza, H., et al. 2010, A\&A 514, 1

[8] La Franca, F., Fiore, F., Comastri, A., et al. 2005, ApJ 635, 864

[9] Lawrence, A., \& Elvis, M. 2010, ApJ 714, 561

[10] Rush, B., Malkan, M.A., \& Spinoglio, L. 1993, A\&AS 89, 1

[11] Schmidt, M. 1968, ApJ 151, 393

[12] Simpson, C. 2005, MNRAS 360, 565

[13] Toba, Y., Oyabu, S., \& Matsuhara H., et al. 2013, PASJ, submitted

[14] Treister, E., Krolik, J.H., \& Dullemond, C. 2008, ApJ 679, 140

[15] Urry, C.M., \& Padovani, P. 1995, PASP 107, 803

[16] Wright, E.L., Eisenhardt, P.R.M., Mainzer, A.K., et al. 2010, AJ 140, 1868 